\section{(1) \\ CrossMark}

\title{
Low-dose corticosteroids during severe community-acquired pneumonia: end of the story
}

\author{
Jean-Damien Ricard ${ }^{1,2,3}$ and Jonathan Messika ${ }^{1,2,3}$
}

Affiliations: ${ }^{1} \mathrm{AP}-\mathrm{HP}$, Service de Réanimation Médico-chirurgicale, Hôpital Louis Mourier, Colombes, France. ${ }^{2}$ INSERM, IAME, 1137, Paris, France. ${ }^{3}$ Univ Paris Diderot, IAME, 1137, Sorbonne Paris Cité, Paris, France.

Correspondence: Jean-Damien Ricard, Service de Réanimation Médico-chirurgicale, Hôpital Louis Mourier, 178 rue des Renouillers, F-92700 Colombes, France. E-mail: jean-damien.ricardalmr.aphp.fr

@ERSpublications

In cases of community-acquired pneumonia, corticosteroids should only be given if septic shock is also present http://ow.ly/FUCHN

Community-acquired pneumonia (CAP) remains a leading cause of death worldwide despite improvement in patient management. Early recognition of lung infection and prompt initiation of adequate antibiotherapy are crucial elements to ensuring favourable outcomes [1-3]. Nonetheless, in a number of cases, death occurs despite both these targets being met. In these patients, possible excessive inflammatory responses, as in sepsis and septic shock, are believed to contribute to unfavourable outcome. Animal models have elegantly shown that part of the inflammatory response, initially destined to combat the pathogens invading the lungs (such as neutrophil products) may induce tissue damage even in the absence of any bacterial challenge [4]. They have also provided evidence that inhibiting inflammatory signalling lessens lung injury in murine models of Escherichia coli [5, 6] or Pseudomonas aeruginosa [7] pneumonia. These results and many others have prompted clinicians to investigate the potential benefit of administering corticosteroids to counterbalance an intense inflammatory process in order to improve outcome. This search has been a long and winding road which many researchers have taken with varying success.

As of 2014, what certainties do we have on the benefits of corticosteroids in the setting of acute respiratory failure?

The ineffectiveness and potential harm of high doses of corticosteroids in the early stage of ARDS might be the only definite conclusion on which many, if not all, clinicians would agree. Regarding sepsis and septic shock, despite more than 30 years' research, few firm conclusions have been reached. If a faster reversal of shock is consistently found with the administration of low-dose corticosteroids, impact on mortality is still a matter of debate $[8,9]$. Results regarding CAP share the same uncertainties. This is perhaps not that surprising since pneumonia is, by far, the leading aetiology of sepsis and septic shock. These uncertainties may reflect the difficulty in differentiating severe CAP from sepsis and septic shock, and providing answers to the following questions: what is severe CAP and when is severe CAP no longer severe CAP but septic shock of pulmonary origin? Does this distinction matter? In other words, is there a specific effect of corticosteroids on CAP, distinct from its effect on septic shock from a pulmonary origin?

In this issue of the European Respiratory Journal, results from TAGAMI et al. [10] shed new light on the potential benefit of corticosteroids and offer an interesting perspective on the current debate. They analysed the data of 6925 patients who received mechanical ventilation for severe CAP in 893 hospitals in Japan between July 2010 and March 2013. Main outcome was 28-day mortality. Authors cogently analysed this large national cohort with respect to catecholamine and corticosteroid uses. Over a third of patients received catecholamine because of associated shock. Among these, another third received corticosteroids.

Received: Dec 082014 | Accepted after revision: Dec 122014

Conflict of interest: Disclosures can be found alongside the online version of this article at erj.ersjournals.com

Copyright OERS 2015 
A propensity score-matched analysis found that 28-day mortality rate was lower in patients who received corticosteroids than in those who did not (propensity score matched: $25.2 \%$ versus $32.6 \%, \mathrm{p}=0.01$ ). In addition, the estimated 28-day mortality reduction in these patients associated with corticosteroids was $27.4 \%$ (95\% CI $12.8-42.1, \mathrm{p}<0.001)$. In the remaining 4401 patients who did not receive catecholamine, a quarter received corticosteroids. Interestingly, their 28-day mortality did not differ from the patients who did not receive steroids (propensity score matched: $17.7 \%$ versus $15.6 \%, \mathrm{p}=0.22$ ). The authors conclude that use of corticosteroids in patients with severe CAP should be limited to those who require catecholamine because of associated septic shock, thereby suggesting that the benefit of corticosteroid therapy is the result of their effect on shock rather than on pneumonia [10].

If the particularly strong design and statistical method used in this study, along with the large number of hospitals and patients analysed, allow us to draw firm conclusions, there are nonetheless a number of limitations that must be recalled. First, important variables are missing such as intensive care unit (ICU) severity scores and pneumonia scores, which would have strengthened internal validity of the results. Second, microbiology data from CAP episodes were not provided, so epidemiological analysis of CAP in these hospitals is not possible, nor is assessment of antibiotic resistance or the virulence of the pathogens involved. This is regrettable considering the noticeable use of antipseudomonal $\beta$-lactam antibiotics, carbapenems and anti-MRSA agents, which are quite uncommon in CAP treatment. Similarly, a quarter of patients with shock received carbapenems, clearly not a first-line molecule for the usually susceptible microorganisms of CAP. One may question whether or not some patients were treated for superinfections or ventilator-acquired pneumonia rather than CAP, thus mixing populations and episodes. Finally, given the size of the study population, potential side-effects of corticosteroids could probably have been identified or ruled out had they been monitored. Nevertheless, we believe that these limitations due to the observational design of the study are outweighed by the sample size and the statistical methods used [11].

How do these results compare with the existing literature? From relative discord, data have gradually converged towards agreement with TAGAm et al. [10]. CONFAlONIERI et al. [12] published a positive randomised controlled trial (RCT) in 46 severe CAP patients assigned to a 7 day, $240 \mathrm{mg}$ per day hydrocortisone treatment or placebo. Despite the small number of patients included, this study evidenced a positive effect of corticosteroids on hospital mortality. However, the external validity of the study is questionable, because of a $0 \%$ mortality in the treatment group. This effect was not found in later studies. MeIJvis et al. [13] randomly assigned 304 non-ICU CAP patients to receive a 4 day, $5 \mathrm{mg}$ dexamethasone treatment or placebo. Although length of stay was 1 day longer in the placebo group $(95 \%$ CI of difference in medians 0-2 days, $\mathrm{p}=0.048$ ), no significant effect was found in in-hospital and 30-day mortality. SNIJDERS et al. [14] randomised 213 hospitalised patients with CAP to receive $40 \mathrm{mg}$ prednisolone for a week or placebo. Again, 30 day mortality did not differ between groups. Finally, a recent meta-analysis pooled nine RCTs evaluating corticosteroids as an adjunctive treatment of CAP [15]. A total of 970 patients were assessable for mortality and use of corticosteroids did not reduce mortality. When analysing the data according to CAP severity, corticosteroids were associated with a reduction in mortality in case of severe CAP but this effect was not found in mixed CAP [15]. Unfortunately, no subgroup analysis was performed according to the presence of shock, and this is probably the crucial point. Mortality in the majority of studies assessing the benefits of corticosteroids in CAP is $\sim 6 \%$, way below that observed in studies on septic shock. For example, mortality in the Corticosteroid Therapy of Septic Shock (CORTICUS) trial was between 31.5\% (placebo group) and 34.3\% (hydrocortisone group) [16]. Results in the present study are in perfect agreement with these rates, as TAGAMI et al. [10] report a $33.4 \% 28$ day mortality rate in patients with shock; not surprisingly, this was significantly greater than that in patients with severe CAP but without shock $(18.5 \%, \mathrm{p}<0.001)$. These results are line with the hypothesis that the beneficial effects of corticosteroids impact the shock component of severe CAP.

If uncertainties remain, believers in corticosteroids may ask the following question: is there any harm in administering low-dose corticosteroids to everyone in order to make sure that those patients who might benefit from this therapy do receive it? Data in the literature argue against such a strategy. First of all, ICU-acquired muscle weakness is a common feature encountered in patients with a prolonged ICU stay treated for severe CAP that requires mechanical ventilation, septic shock or acute respiratory distress syndrome. Main risk factors for this debilitating complication include the use of corticosteroids and the use of neuromuscular blocking agents, which are often associated in the management of the above-mentioned situations $[17,18]$. Second, the risk of superinfection is not null and more episodes of superinfection, including new sepsis and septic shock, were observed in the hydrocortisone group of the CORTICUS study [16]. Another reason for refraining from administering low-dose corticosteroids to all patients with acute respiratory failure possibly due to lung infection is the detrimental effect of corticosteroids in case of severe influenza-related respiratory distress. Results from several cohort studies $[19,20]$, indicate no benefit from corticosteroids administration and even worse, suggest either increased 
mortality [19] or increased risk of superinfection [20]. These negative results were confirmed in a meta-analysis reporting increased odds of mortality (OR 2.12, 95\%CI 1.36-3.29) associated with corticosteroid treatment [21].

Finally, one must bear in mind the potential detrimental effect of blunting the inflammatory response on the infectious process. This has recently been clearly evidenced with the use of other potent anti-inflammatory treatments such as nonsteroidal anti-inflammatory drugs (NSAIDs) during CAP. Indeed, we [22] and others [23] have found that patients exposed to NSAIDs during the early stage of CAP had a worse presentation of CAP, more pleuropulmonary complications and required noninvasive ventilatory support more often, such as high-flow oxygen therapy [22]. It is worth noting that in the study by TAGAMI et al. [10] study, NSAIDs were given to $\sim 10 \%$ of the whole study population, irrespective of the use of corticosteroids. The question of whether the pleuropulmonary complications reported were related to the use of NSAIDs remains unanswered.

Thanks to TAGAMI et al. [10], we now have a clearer view of the benefits of corticosteroids during CAP and which patients should receive them. Positive effects of corticosteroids during severe CAP are related to the beneficial effects of corticosteroids on septic shock. Thus, patients with severe CAP should not receive corticosteroids, unless shock that requires vasopressor infusion is present. In addition, corticosteroids should not be given in case of influenza-related respiratory distress [21] and general practitioners should refrain from giving NSAID to patients in the early stage of CAP [22].

\section{References}

1 Menendez R, Torres A, Reyes S, et al. Initial management of pneumonia and sepsis: factors associated with improved outcome. Eur Respir J 2012; 39: 156-162.

2 Waterer GW, Rello J, Wunderink RG. Management of community-acquired pneumonia in adults. Am J Respir Crit Care Med 2011; 183: 157-164.

3 Gattarello S, Borgatta B, Sole-Violan J, et al. Decrease in mortality in severe community-acquired pneumococcal pneumonia: impact of improving antibiotic strategies (2000-2013). Chest 2014; 146: 22-31.

4 Cheng DS, Han W, Chen SM, et al. Airway epithelium controls lung inflammation and injury through the NF- $\mathrm{B}$ pathway. J Immunol 2007; 178: 6504-6513.

5 Mizgerd JP, Lupa MM, Hjoberg J, et al. Roles for early response cytokines during Escherichia coli pneumonia revealed by mice with combined deficiencies of all signaling receptors for TNF and IL-1. Am J Physiol 2004; 286 : L1302-L1310.

6 Mizgerd JP, Spieker MR, Doerschuk CM. Early response cytokines and innate immunity: essential roles for TNF receptor 1 and type I IL-1 receptor during Escherichia coli pneumonia in mice. J Immunol 2001; 166: $4042-4048$.

7 Gibot S, Alauzet C, Massin F, et al. Modulation of the triggering receptor expressed on myeloid cells-1 pathway during pneumonia in rats. J Infect Dis 2006; 194: 975-983.

8 Allen KS, Kinasewitz GT. The pendulum of corticosteroids in sepsis swings again? Crit Care Med 2014; 42: 2442-2443.

9 Funk D, Doucette S, Pisipati A, et al. Low-dose corticosteroid treatment in septic shock: a propensity-matching study. Crit Care Med 2014; 42: 2333-2341.

10 Tagami T, Matsui H, Horiguchi H, et al. Low-dose corticosteroid use and mortality in severe community-acquired pneumonia patients. Eur Respir J 2014; 45: 463-472.

11 Borah BJ, Moriarty JP, Crown WH, et al. Applications of propensity score methods in observational comparative effectiveness and safety research: where have we come and where should we go? J Comp Eff Res 2014; 3: 63-78.

12 Confalonieri M, Urbino R, Potena A, et al. Hydrocortisone infusion for severe community-acquired pneumonia: a preliminary randomized study. Am J Respir Crit Care Med 2005; 171: 242-248.

13 Meijvis SC, Hardeman H, Remmelts HH, et al. Dexamethasone and length of hospital stay in patients with community-acquired pneumonia: a randomised, double-blind, placebo-controlled trial. Lancet 2011; 377: 2023-2030.

14 Snijders D, Daniels JM, de Graaff CS, et al. Efficacy of corticosteroids in community-acquired pneumonia: a randomized double-blinded clinical trial. Am J Respir Crit Care Med 2010; 181: 975-982.

15 Nie W, Zhang Y, Cheng J, et al. Corticosteroids in the treatment of community-acquired pneumonia in adults: a meta-analysis. PLoS One 2012; 7: e47926.

16 Sprung CL, Annane D, Keh D, et al. Hydrocortisone therapy for patients with septic shock. N Engl J Med 2008; 358: $111-124$.

17 De Jonghe B, Sharshar T, Lefaucheur JP, et al. Paresis acquired in the intensive care unit: a prospective multicenter study. JAMA 2002; 288: 2859-2867.

18 De Jonghe B, Lacherade JC, Sharshar T, et al. Intensive care unit-acquired weakness: risk factors and prevention. Crit Care Med 2009; 37: Suppl., S309-S315.

19 Brun-Buisson C, Richard JC, Mercat A, et al. Early corticosteroids in severe influenza A/H1N1 pneumonia and acute respiratory distress syndrome. Am J Respir Crit Care Med 2011; 183: 1200-1206.

20 Martin-Loeches I, Lisboa T, Rhodes A, et al. Use of early corticosteroid therapy on ICU admission in patients affected by severe pandemic (H1N1)v influenza A infection. Intensive Care Med 2011; 37: 272-283.

21 Rodrigo C, Leonardi-Bee J, Nguyen-Van-Tam JS, et al. Effect of corticosteroid therapy on influenza-related mortality: a systematic review and meta-analysis. J Infect Dis 2014 [In press DOI: 10.1093/infdis/jiu645].

22 Messika J, Sztrymf B, Bertrand F, et al. Risks of nonsteroidal antiinflammatory drugs in undiagnosed intensive care unit pneumococcal pneumonia: younger and more severely affected patients. J Crit Care 2014; 29: 733-738.

23 Voiriot G, Dury S, Parrot A, et al. Nonsteroidal antiinflammatory drugs may affect the presentation and course of community-acquired pneumonia. Chest 2011; 139: 387-394. 